\title{
Non-Local Dispersal and Bistability
}

\author{
V. Hutson \\ Department of Applied Mathematics \\ The University of Sheffield \\ Sheffield S3 7RH, U.K.
}

\author{
M. Grinfeld \\ Department of Mathematics \\ University of Strathclyde \\ Glasgow G1 1XH, U.K.
}

December 15, 2004

\begin{abstract}
The scalar initial value problem

$$
u_{t}=\rho D u+f(u),
$$

is a model for dispersal. Here $u$ represents the density at point $x$ of a compact spatial region $\Omega \in \mathbb{R}^{n}$ and time $t$, and $u(\cdot)$ is a function of $t$ with values in some function space $B$. $D$ is a bounded linear operator and $f(u)$ is a bistable nonlinearity for the associated ODE $u_{t}=f(u)$. Problems of this type arise in mathematical ecology and materials science where the simple diffusion model with $D=\Delta$ is not sufficiently general. The study of the dynamics of the equation presents a difficult problem which crucially differs from the diffusion case in that the semiflow generated is not compactifying. We study the asymptotic behaviour of solutions and ask under what conditions each positive semi-orbit converges to an equilibrium (as in the case $D=\Delta$ ). We develop a technique for proving that indeed convergence does hold for small $\rho$ and show by constructing a counter-example that this result does not hold in general for all $\rho$.
\end{abstract}

\section{Introduction}

The present investigation is motivated by a class of models of non-local spatial dispersal in which the dispersal operator $D$, say, involves an integral operator. For example

$$
D u(x)=\int_{0}^{1} k(x, y)[u(y)-u(x)] d y .
$$

Such models occur in the theory of phase transition, ecology, genetics and neurology; for further details and references see section 2. We consider here the scalar case with governing equation

$$
u_{t}=\rho D u+f(u),
$$

where $u: \Omega \times[0, \infty) \rightarrow \mathbb{R}$, and the suffix $t$ represents differentiation. Here $\Omega \subset \mathbb{R}^{n}$ is a bounded spatial region, the parameter $\rho$ is the dispersal strength, and $f$ is the reaction term. 
There is now a considerable body of theory on this class of equation. However, there remain several fundamental open questions even for the scalar case, among which those concerning the asymptotic behaviour for a multi-stable reaction term are of particular mathematical interest. To fix ideas let us suppose that $f(u)$ is the cubic, $f(u)=u\left(1-u^{2}\right)$. Then for the corresponding ordinary differential equation (ODE) with $u: \mathbb{R} \rightarrow \mathbb{R}$

$$
u_{t}=u\left(1-u^{2}\right),
$$

\pm 1 are attractors while 0 is a repellor. An analogous case, where $D=\Delta$, the Laplacian, and zero Neumann conditions are imposed, is a classical problem (PDE), which has been much studied, see for example [2, 8, 12, 16].

The analysis of the asymptotic behaviour depends crucially on the compactifying action of the semi-flow, and it is here that the present problem differs fundamentally, as this property no longer holds. In the PDE case it is known that all orbits approach an equilibrium. It is the purpose of this paper to show that an analogous result, with pointwise convergence, holds in the present case for small $\rho$.

The bistable case has been studied from this point of view in [4, 9, 17]. It is known [4, 17] that for small $\rho$, equation (1.2) has an uncountable set of equilibria in $L^{\infty}$, almost all of which are not continuous. Thus there are difficulties with proving compactness of the global attractor in $L^{p}$ spaces. In [9] it was shown that for large $\rho$, for a class of kernel leading to the analogue of the above PDE case (see Example 2.1), the asymptotic behaviour follows that of ODE, with results analogous to [3] for systems of PDEs, being obtained. A similar problem is considered in [5] under the technical condition $n \leq 3$; using rather sophisticated techniques it is shown [5, Theorem 1.2] that in the case when all stationary solutions of the governing equation are continuous, all orbits converge in $L^{2}$ to a stationary solution. This restriction means that the result does not cover cases when $\rho$ is not large. When $k$ is a constant, it was proved that for all $\rho$, for a wide class of initial condition, there is pointwise convergence to an equilibrium. The argument depended on being able to show that the positive semi-orbit is relatively compact. However, this argument has no obvious extension to general $k$, and it is unknown at the present time whether, and under what conditions, convergence holds; this question is further discussed in section 4 .

Here we follow a completely different approach, which we hope will be useful for a wide range of problems with rather general dispersal terms. No compactness either of the operator $K$ nor of the semi-flow generated by $D$ can be assumed, so the standard methods for PDE are not available. At first sight there seems to be a possibility of using the variation of parameters formula for the operator $\partial / \partial t-\rho D$ if $\rho$ is small, but it is not clear how the argument would go, and the literature does not appear to give much guidance. Here, then, we present a method, essentially based on a regular perturbation of the whole semi-orbit. This applies for quite general $D$ if $\rho$ is small.

The background is outlined in section 2, and the main convergence result is given in section 3 . In section 4 possible extensions are examined and it is noted that convergence to an equilibrium cannot hold for all $\rho$ and general $K$ since a Hopf bifurcation may occur. However, it is conjectured that for a restricted class of $K$, say an integral operator with a positive symmetric 
kernel $k$, the result given in [9] for $k=1$ may generalise. Finally we suggest that the current argument may extend to a whole class of multi-stable systems.

\section{Basic assumptions}

The aim is to treat a dispersal operator $D$ satisfying only weak assumptions. The reaction term $f$ assumed leads to a bistable system, but the analysis would easily extend to an $f$ with multiple zeros, so long as these are hyperbolic.

With compact $\Omega \subset \mathbb{R}^{n},\|\cdot\|_{p}$ is the $L^{p}$ norm for functions $\Omega \rightarrow \mathbb{R}$. $C^{k}(\mathbb{R})$ will be the $k$ times continuously differentiable functions $\mathbb{R} \rightarrow \mathbb{R}$. For functions $\Omega \times \mathbb{R}^{+} \rightarrow \mathbb{R}$, the suffix ' $t$ ' will denote differentiation with respect to time $t$ with the space variable $x \in \Omega$ fixed. $m$ is Lebesgue measure on $\mathbb{R}^{n}$.

The governing equation is the following initial value problem:

$$
u_{t}=f(u)+\rho(K u-B u)
$$

where $u: \Omega \times \mathbb{R}^{+} \rightarrow \mathbb{R}$, and $u(x, 0)=u_{0}(x),(x \in \Omega)$, is given. Here $B$ is a multiplication operator:

$$
B u(x)=b(x) \cdot u(x),
$$

and $K$ is a linear bounded operator; precise conditions on $B$ and $K$ are given in $H 1$ below. The linear dispersal operator $D=K-B$, and the dispersal rate is $\rho \geq 0$. Two examples, which have been extensively discussed, are as follows.

Example 2.1. An integral dispersal operator, arising in the biological context, has been considered in $[11,9,10,15]$. One assumes for example that

$$
D u(x)=\int_{\Omega} k(x, y) u(y) d y-u(x),
$$

so here $B=I$ and

$$
K u(x)=\int_{\Omega} k(x, y) u(y) d y .
$$

Often $k$ is taken to be positive and $\int_{\Omega} k(x, y) d y \leq 1$. This last condition means that no new individuals are created by the dispersal. In particular, there is a class of kernel, discussed in [11], leading to a condition of no dispersal across the boundary, which yields the analogue of the PDE case $D=\Delta$ with zero Neumann boundary conditions. In an interesting neurological model, see [14], $k$ need not be positive.

Example 2.2.

$$
D u(x)=\int_{\Omega} k(x, y)[u(y)-u(x)] d y,
$$


where $K$ is as before and

$$
B u(x)=u(x) \int_{\Omega} k(x, y) d y .
$$

This model has been commonly used in the theory of phase transitions, see $[6,7,4]$ and the numerous references therein.

Conditions $\mathrm{H} 1$ and $\mathrm{H} 2$ below will be assumed throughout.

H1(a) $\Omega$ is compact with $m(\Omega)=1$.

(b) $b: \Omega \rightarrow \mathbb{R}$ is continuous and $|b(x)| \leq 1 \quad(x \in \Omega)$.

(c) The linear operator $K: L^{1}(\Omega) \rightarrow L^{\infty}(\Omega)$ is bounded with bound 1:

$$
\|K u\|_{\infty} \leq\|u\|_{1} \quad\left(u \in L^{1}\right) .
$$

H2(a) $f \in C^{2}(\mathbb{R})$.

(b) $f$ has exactly 3 zeros $\alpha_{1}, \alpha_{2}, \alpha_{3}$ with $\alpha_{1}<\alpha_{2}<\alpha_{3}$ and $\left|\alpha_{i}\right|<1 \quad(i=1,3)$.

(c) $f^{\prime}\left(\alpha_{1}\right)<0, f^{\prime}\left(\alpha_{2}\right)>0, f^{\prime}\left(\alpha_{3}\right)<0$.

The two conditions $m(\Omega)=1$ and $\left|\alpha_{i}\right|<1$ in $\mathrm{H} 1$ (a) and $\mathrm{H} 2(\mathrm{~b})$, respectively, simplify the notation and of course only involve rescalings of the space variable $x$ and of $u$ respectively.

The following simple observation enables us to construct a positively invariant region. From $\mathrm{H} 2$, there is an interval, say $I=\left[l_{1}, l_{2}\right]$, with

$$
\left|l_{1}\right|,\left|l_{2}\right| \leq 1
$$

such that $\alpha_{i} \in\left(l_{1}, l_{2}\right), \quad(i=1,2,3)$, and such that for some $\eta>0$,

$$
f\left(l_{2}\right) \leq-\eta, \quad f\left(l_{1}\right) \geq \eta .
$$

Definition 2.3. Let $Z$ be the set of measurable functions $u: \Omega \rightarrow \mathbb{R}$ such that

$$
\left.l_{1} \leq u(x) \leq l_{2} \quad \text { (for a.a. } x \in \Omega\right) .
$$

Equip $Z$ with the metric $d$ induced by the $L^{1}$ norm.

It will be useful later to note that, since $m(\Omega)=1$, from (2.3) and (2.4), for $u \in Z$,

$$
\|K u\|_{\infty} \leq\|u\|_{1} \leq\|u\|_{\infty} .
$$

Lemma 2.4. There exists $\rho_{0}>0$ such that for $0 \leq \rho \leq \rho_{0}$, the initial value problem (2.1) generates a semi-flow on $Z$. 
Proof. The global existence of orbits is proved in $[4,17]$. For the invariance, from H1 (b), (c), for $x \in \Omega$,

$$
\begin{aligned}
|D u(x)| & \leq 2\|u\|_{\infty}, \\
& \leq 2 \max \left(\left|l_{1}\right|,\left|l_{2}\right|\right), \\
& \leq 2
\end{aligned}
$$

from (2.4). Hence from (2.5),

$$
\begin{aligned}
u_{t} & =f(u(x, t))+\rho D u(x, t), \\
& \leq-\eta+2 \rho_{0}
\end{aligned}
$$

if $u(x, t)=l_{2}$. Thus $u_{t}<0$ if $u=l_{2}$ and $\rho_{0}$ is small enough. A similar argument holds if $u=l_{1}$, and the invariance follows.

For the dissipativity of (2.1), a slight strengthening of $\mathrm{H} 2$ is probably needed, but we shall not consider this issue here, and the analysis will henceforth be restricted to $Z$.

\section{Convergence to an equilibrium}

It will be shown in Theorem 3.5 that for small enough $\rho$, every solution of (2.1) converges in $L^{1}$ to an equilibrium; it is then an easy corollary that the convergence is pointwise. The argument is intuitively straightforward and is based on being able to exploit the broad idea that (2.1) is a 'nice' perturbation of the corresponding family of ordinary differential equations $u_{t}=f(u)$ for each $x$.

A simple lemma concerning the perturbation of the roots of an equation in $\mathbb{R}$ is needed. For $z, v, b \in \mathbb{R}$ define

$$
E_{\rho}(z)=f(z)+\rho(v-b z) .
$$

We have $E_{0}(z)=f(z)$, and from $\mathrm{H} 2$, the roots, $\alpha_{i}(\rho)$ say, of $E_{\rho}(z)=0$ depend smoothly on $\rho$ for small $\rho$, and are thus close to the $\alpha_{i}(0)=\alpha_{i}$. It is thus easy to establish the next lemma using a Taylor series. Broadly, the lemma provides a means of using the hyperbolicity to study the simple properties of the perturbed vector field.

Lemma 3.1. There exist $\rho_{0}>0, \varepsilon_{0}>\rho_{0}, c>0$ such that the following holds. Given $\varepsilon \in$ $\left(0, \varepsilon_{0}\right], \rho \in\left[0, \rho_{0}\right]$, there exists $\delta>0$ such that for all $v, b$ with $|v| \leq 1,|b| \leq 1$,

$$
\begin{aligned}
& E_{\rho}(z)>0 \quad\left(l_{1} \leq z \leq \alpha_{1}(\rho)-c \varepsilon\right), \\
& E_{\rho}(z)<0 \quad\left(\alpha_{1}(\rho)+c \varepsilon \leq z \leq \alpha_{2}(\rho)-c \varepsilon\right), \\
& E_{\rho}(z)>0 \quad\left(\alpha_{2}(\rho)+c \varepsilon \leq z \leq \alpha_{3}(\rho)-c \varepsilon\right), \\
& E_{\rho}(z)<0 \quad\left(\alpha_{3}(\rho)+c \varepsilon \leq z \leq l_{2}\right),
\end{aligned}
$$

and in each of these intervals,

$$
\left|E_{\rho}(z)\right|-\varepsilon \geq \delta .
$$


As subsequently $\rho$ will be fixed and $b$ will be a fixed function, it is appropriate to modify the notation and for $v, z: \Omega \rightarrow \mathbb{R}$ define

$$
E(v(x), z(x), x)=f(z(x))-\rho b(x) \cdot z(x)+\rho v(x) .
$$

It is often convenient to contract the notation and to write $E(v, z, x)$, the meaning being clear from the context. For given $v(x)$, let $z_{i}(x),(i=1,2,3)$, with $z_{1}(x)<z_{2}(x)<z_{3}(x)$ denote the solutions of the equation

$$
E(v(x), z(x), x)=0 .
$$

The governing equation (2.1) becomes

$$
u_{t}=E(K u, u, x)
$$

In order to tackle this, we consider the initial value problem

$$
u_{t}=E(v(x), u(x, t), x)+\varepsilon g(x, t)
$$

for $\tau \geq 0$ with $u(x, \tau) \in Z$ given. We wish to understand the behaviour of $u$ for small $\rho$ and $\varepsilon$; the following result shows how the dynamics are qualitatively inherited from the $\rho=0$ case.

Proposition 3.2. Assume that

$$
\begin{aligned}
|v(x)| & \leq 1 \quad(x \in \Omega), \\
|g(x, t)| & \leq 1 \quad(x \in \Omega, t \geq \tau)
\end{aligned}
$$

Then there exist $\varepsilon_{0}, \rho_{0}, c$ with

(a) $0<\rho_{0}<\varepsilon_{0}$,

(b) $0<c \rho_{0}<1 / 2$,

such that the following holds. Take any $\rho \in\left[0, \rho_{0}\right], \varepsilon \in\left(0, \varepsilon_{0}\right]$, and assume that $u$ satisfies (3.10) with $u(x, \tau) \in Z$. Then there exist $T \geq \tau, \tilde{u} \in Z$, and $\hat{u}$ such that

$$
u(x, t)-\tilde{u}(x)=2 \varepsilon c \hat{u}(x, t),
$$

where

(i) $E(v(x), \tilde{u}(x), x)=0 \quad(x \in \Omega)$,

(ii) $\|\hat{u}(\cdot, t)\|_{1} \leq 1 \quad(t \geq T)$.

To clarify this result, we define disjoint subsets $\Omega_{i}(t)$ of $\Omega$ with $\cup \Omega_{i}(t)=\Omega$. The definition is written down explicitly for $\Omega_{i}^{+}(t)(i=2,3,4)$, with $u(x, t)>z_{2}(x)$; the definitions of $\Omega_{i}^{-}(t)$ are analogous. Recall that the interval $I=\left[l_{1}, l_{2}\right]$ is invariant.

$$
\Omega_{1}(t)=\left\{x: z_{2}(x)-c \varepsilon<u(x, t)<z_{2}(x)+c \varepsilon\right\},
$$




$$
\begin{aligned}
\Omega_{2}^{+}(t) & =\left\{x: z_{2}(x)+c \varepsilon \leq u(x, t) \leq z_{3}(x)-c \varepsilon\right\}, \\
\Omega_{3}^{+}(t) & =\left\{x: z_{3}(x)-c \varepsilon<u(x, t)<\min \left[l_{2}, z_{3}(x)+c \varepsilon\right]\right\}, \\
\Omega_{4}^{+}(t) & =\left\{x: z_{3}(x)+c \varepsilon \leq u(x, t) \leq l_{2}\right\}, \\
\Omega_{j}(t) & =\Omega_{j}^{+}(t) \cup \Omega_{j}^{-}(t) \quad(i=2,3,4) .
\end{aligned}
$$

Thus $\Omega_{1}(t), \Omega_{3}(t)$ are sets where $u(x, t)$ is 'near' an equilibrium of the reaction system, whereas in $\Omega_{2}(t), \Omega_{4}(t)$ this is not the case. The intuitive idea behind Proposition 3.2 is that from (3.2) to (3.6), for fixed $x \in \Omega, u(x, t)$ can only remain for a uniformly (in $x$ ) limited time outside a small neighbourhood of an equilibrium.

We use the notation $\nearrow, \searrow$ for 'increasing' and 'decreasing' respectively in the sense of set inclusion, without the implication of 'strict'.

Lemma 3.3. The following hold under the conditions of Proposition 3.2.

(a) $\Omega_{1}(\cdot) \searrow, \Omega_{3}(\cdot) \nearrow$.

(b) $m\left(\Omega_{2}(t)\right), m\left(\Omega_{4}(t)\right) \rightarrow 0$ as $t \rightarrow \infty$.

(c) Define sets $\Omega_{1}(\infty), \Omega_{3}(\infty)$ (unique up to sets of measure zero) with the properties:

$$
\begin{aligned}
\Omega_{1}(t) \supset \Omega_{1}(\infty) \quad(t \geq \tau), \quad \lim _{t \rightarrow \infty} m\left(\Omega_{1}(t)\right)=m\left(\Omega_{1}(\infty)\right) \\
\Omega_{3}^{ \pm}(t) \subset \Omega_{3}^{ \pm}(\infty) \quad(t \geq \tau), \quad \lim _{t \rightarrow \infty} m\left(\Omega_{3}^{ \pm}(t)\right)=m\left(\Omega_{3}^{ \pm}(\infty)\right),
\end{aligned}
$$

respectively. Then

$$
m\left(\Omega_{1}(\infty)+m\left(\Omega_{3}(\infty)\right)=1\right.
$$

Proof. From (3.10) and (3.12),

$$
u_{t}(x, t) \geq E(v(x), u(x, t), x)-\varepsilon .
$$

Hence, from Lemma 3.1, (3.3) and (3.4) respectively, and (3.6), there exists $\delta>0$ such that

$$
\begin{aligned}
& u_{t}(x, t) \leq-\delta\left(x \in \Omega_{2}^{-}(t)\right), \\
& u_{t}(x, t) \geq \delta\left(x \in \Omega_{2}^{+}(t)\right) .
\end{aligned}
$$

If $x \in \Omega_{1}\left(t_{0}\right)$ for some $t_{0}$, either this holds for all $t>t_{0}$, or $x \in \Omega_{2}\left(t_{1}\right)$ for some $t_{1}>t_{0}$. Then from (3.15) and (3.16), $x \notin \Omega_{1}(t)$ for $t \geq t_{1}$. Therefore $\Omega_{1}(\cdot) \searrow$. Analogous arguments complete the proof of (a).

The following is next established:

$$
\lim _{t \rightarrow \infty} m\left(\Omega_{2}^{+}(t)\right)=0
$$

Since (by assumption) $l_{2}-l_{1} \leq 2$, from (3.16),

$$
x \in \Omega_{2}^{+}\left(t_{0}\right) \Rightarrow x \in \Omega_{3}^{+}(t) \quad\left(t>t_{0}+2 \delta^{-1}\right) .
$$


If (3.17) does not hold, there exists a sequence $\left\{t_{n}\right\} \rightarrow \infty$, and an $\eta>0$ such that $m\left(\Omega_{2}^{+}\left(t_{n}\right)\right) \geq$ $\eta(\forall n)$. Choose an infinite subsequence, still denoted by $\left\{t_{n}\right\}$ such that

$$
t_{n}-t_{n-1}>2 \delta^{-1}
$$

Then from (3.18)

$$
\Omega_{2}^{+}\left(t_{n}\right) \cap \Omega_{2}^{+}\left(t_{i}\right)=\emptyset \quad(i \neq n) .
$$

Therefore

$$
\sum_{j=1}^{n} m\left(\Omega_{2}^{+}\left(t_{j}\right)\right) \geq n \eta .
$$

However, this leads to a contradiction for $n$ large enough, as $m(\Omega)<\infty$. This proves (3.17). An analogous argument gives the corresponding result for $\Omega_{2}^{-}(t)$, and so the first claim in (b). A similar argument yields the second part of (b). (c) is an obvious consequence.

Proof of Proposition 3.2. Define

$$
\tilde{u}(x)= \begin{cases}z_{1}(x) & \left(x \in \Omega_{3}^{-}(\infty)\right), \\ z_{2}(x) & \left(x \in \Omega_{1}(\infty)\right), \\ z_{3}(x) & \left(x \in \Omega_{3}^{+}(\infty)\right) .\end{cases}
$$

By definition of the $\Omega_{i}(t)$,

$$
|u(x, t)-\tilde{u}(x)|<c \varepsilon
$$

for $x \in \Omega_{1}(t) \cup \Omega_{3}(t)$. From Lemma 3.3(b), for large enough $t$,

$$
m\left(\Omega_{2}(t) \cup \Omega_{4}(t)\right)<c \varepsilon .
$$

Since $|u(x, t)| \leq 1$ for all $x$ and $t$, the result follows.

Given $u(x, 0)$, the construction above leads to unique $\tilde{u}$ and $\hat{u}$. To explain the argument used in the proof of the next lemma, we introduce the somewhat cumbersome notation $P(\tau, v, \varepsilon, g)$ for the initial value problem 3.10, with $\tilde{u}=\tilde{U}(\tau, v, \varepsilon, g), \hat{u}=\hat{U}(\tau, v, \varepsilon, g)$ in Proposition 3.2.

Lemma 3.4 below shows how Proposition 3.2 may be used iteratively to improve the approximation to the solution of the governing equation (2.1). In terms of the semi-flow, we are showing that for large $t$ approximations to the positive orbit, uniform in $t$, may be obtained.

If $\rho=0$, it is of course a triviality to prove the main result, that is the $L^{1}$ convergence to an equilibrium, Theorem 3.5 below. However, since the approach to this theorem is via a perturbation in $\rho$, we check that Proposition 3.2 gives the result if $\rho=0$. For we may take $\rho=0, g=0$ and the result will follow by choosing, for example, a sequence $\left\{\varepsilon_{n}\right\} \rightarrow 0$. It will indeed be assumed, for convenience in the notation, that $\rho>0$ in the proof of Lemma 3.4 below.

Lemma 3.4. There exist $\rho_{0}>0, c>0$ with $2 \rho_{0} c<1$ such that the following holds. Fix any $\rho \in\left[0, \rho_{0}\right]$ and any $u_{0} \in Z$. Let $u$ be the (unique) global solution of (2.1). Then there exist sequences $\left\{t^{(n)}\right\},\left\{\tilde{u}^{(n)}(x)\right\},\left\{\hat{u}^{(n)}(x, t)\right\}$ such that 


$$
u(x, t)-\tilde{u}^{(n)}(x)=(2 \rho c)^{n} \hat{u}^{(n)}(x, t) \quad\left(n \geq 1, t \geq t^{(n)}\right),
$$

with

$$
\tilde{u}^{(0)}=0, \quad \hat{u}^{(0)}=u,
$$

where

$$
E\left(K \tilde{u}^{(n-1)}, \tilde{u}^{(n)}, x\right)=0
$$

and

$$
\left\|\hat{u}^{(n)}(\cdot, t)\right\|_{1} \leq 1
$$

Proof. Recall from a previous remark that we are assuming that $\rho>0$. The argument is by induction, the first step being as follows. Rewrite (2.1) in the form

$$
u_{t}=E\left(K \tilde{u}^{(0)}, u, x\right)+\rho g^{(0)}(x, t)
$$

where $t^{(0)}=0, \tilde{u}^{(0)}=0, \hat{u}^{(0)}=u$ and $g^{(0)}=K \hat{u}^{(0)}=K u$. This gives the initial value problem $P\left(0, K \tilde{u}^{(0)}, \rho, K \hat{u}^{(0)}\right)$. Since $\|u\|_{1} \leq 1$, from (2.7),

$$
\left\|g^{(0)}(\cdot, t)\right\|_{\infty} \leq 1 \quad\left(t \geq t^{(0)}\right)
$$

In Proposition 3.2, take $v=0, \varepsilon=\rho$, obtaining for some $t^{(1)}$,

$$
u(x, t)-\tilde{u}^{(1)}(x)=(2 c \rho) \hat{u}^{(1)}(x, t) \quad\left(t \geq t^{(1)}\right),
$$

where

$$
E\left(K \tilde{u}^{(0)}, \tilde{u}^{(1)}, x\right)=0
$$

and

$$
\left\|\hat{u}^{(1)}(\cdot, t)\right\|_{1} \leq 1
$$

Thus (3.19), (3.20), (3.21) hold with $n=1$. Here

$$
\tilde{u}^{(1)}=\tilde{U}\left(0, K \tilde{u}^{(0)}, \rho, K \hat{u}^{(0)}\right), \quad \hat{u}^{(1)}=\hat{U}\left(0, K \tilde{u}^{(0)}, \rho, K \hat{u}^{(0)}\right) .
$$

Now assume that (3.19)-(3.21) hold for $j \geq 1$, and define

$$
\begin{aligned}
& v^{(j)}=K \tilde{u}^{(j)}, \\
& g^{(j)}=K \hat{u}^{(j)} .
\end{aligned}
$$

From (2.7),

$$
\left\|v^{(j)}(\cdot)\right\|_{\infty} \leq 1
$$

since $\tilde{u}^{(j)} \in Z$, and

$$
\left\|g^{(j)}(\cdot, t)\right\|_{\infty} \leq 1 \quad\left(t \geq t^{(j)}\right)
$$

since (3.21) holds. Now substitute (3.19) with $n=j$ into (2.1) obtaining

$$
u_{t}=E\left(K \tilde{u}^{(j)}, u, x\right)+\rho(2 \rho c)^{j} g^{(j)} .
$$


This gives the initial value problem $P\left(t^{(j)}, K \tilde{u}^{(j)},(2 \rho c)^{j}, K \hat{u}^{(j)}\right)$. We apply Proposition 3.2 again with $v=v^{(j)}, g=g^{(j)}, \varepsilon=\rho(2 \rho c)^{j}$, and deduce that for some $t^{(j+1)}$,

$$
u(x, t)-\tilde{u}^{(j+1)}(x)=(2 \rho c)^{j+1} \hat{u}^{(j+1)}(x, t) \quad\left(t \geq t^{(j+1)}\right),
$$

where

$$
\begin{gathered}
E\left(K \tilde{u}^{(j)}, \tilde{u}^{(j+1)}, x\right)=0, \\
\left\|\hat{u}^{(j+1)}(\cdot, t)\right\|_{1} \leq 1 .
\end{gathered}
$$

Here

$$
\begin{aligned}
& \tilde{u}^{(j+1)}=\tilde{U}\left(t^{(j)}, K \tilde{u}^{(j)},(2 \rho c)^{j}, K \hat{u}^{(j)}\right), \\
& \hat{u}^{(j+1)}=\hat{U}\left(t^{(j)}, K \tilde{u}^{(j)},(2 \rho c)^{j}, K \hat{u}^{(j)}\right) .
\end{aligned}
$$

Thus (3.19)-(3.21) hold with $n=j+1$. The usual induction argument completes the proof.

Theorem 3.5. Choose any $\rho \in\left[0, \rho_{0}\right]$ and $u_{0} \in Z$. Then there is an equilibrium $\tilde{u}$ of (2.1) in $Z$ such that $u(t) \rightarrow \tilde{u}$ in $L^{1}$ as $t \rightarrow \infty$.

Proof. From (3.19),

$$
\tilde{u}^{(n)}+(2 \rho c)^{n} \hat{u}^{(n)}=\tilde{u}^{(m)}+(2 \rho c)^{m} \hat{u}^{(m)},
$$

where from (3.21), for $t \geq \max \left(t^{(n)}, t^{(m)}\right)$,

$$
\left\|\hat{u}^{(j)}(\cdot, t)\right\|_{1} \leq 1 \quad(j=n, m) .
$$

Thus, since $2 \rho c<1,\left\{\tilde{u}^{(n)}\right\}$ is a Cauchy sequence in $Z$; since $Z$ is complete, the sequence has a limit $\tilde{u}$, say, in $Z$. It follows from the continuity of $E$ on $Z$ that $\tilde{u}$ is an equilibrium.

To prove the convergence, assume the contrary. Then there is a sequence $\left\{t^{(k)}\right\} \rightarrow \infty$ and $\varepsilon>0$ such that

$$
\left\|u\left(\cdot, t^{(k)}\right)-\tilde{u}(\cdot)\right\|_{1} \geq \varepsilon \quad(\forall k) .
$$

Since $\tilde{u}^{(k)} \rightarrow \tilde{u}$ as $k \rightarrow \infty$, there exists $k_{1}$ such that

$$
\left\|\tilde{u}^{(k)}-\tilde{u}\right\|_{1} \leq \varepsilon / 4 \quad\left(k \geq k_{1}\right) .
$$

Choose $k_{2}$ such that

$$
(2 \rho c)^{k} \leq \varepsilon / 4 \quad\left(k \geq k_{2}\right),
$$

and set $k_{3}=\max \left(k_{1}, k_{2}\right)$. From (3.19)

$$
u(x, t)-\tilde{u}(x)=\tilde{u}^{(k)}(x)-\tilde{u}(x)+(2 \rho c)^{k} \hat{u}^{(k)}(x, t) .
$$

Then for all $k \geq k_{3}$, from (3.21),

$$
\begin{aligned}
\left\|u\left(\cdot, t^{(k)}\right)-\tilde{u}(\cdot)\right\|_{1} & \leq\left\|\tilde{u}^{(k)}-\tilde{u}\right\|_{1}+(2 \rho c)^{k}, \\
& \leq \varepsilon / 2
\end{aligned}
$$


from (3.23) and (3.24). This contradicts (3.22).

Corollary 3.6. The convergence is pointwise, that is there is a $\tilde{u} \in Z$ such that

$$
\lim _{t \rightarrow \infty} u(x, t)=\tilde{u}(x) \quad(x \in \Omega) .
$$

Proof. By Theorem 3.5 and H1(c), there is a $v \in L^{\infty}(\Omega)$ such that, with convergence in $L^{1}$,

$$
\lim _{t \rightarrow \infty} K u(t)=v
$$

Hence, for any fixed $x \in \Omega,(2.1)$ may be written as the non-autonomous ordinary differential equation

$$
\dot{u}=f(u)+\rho(v(x)-b(x) u)+h(x, t)
$$

where $\lim _{t \rightarrow \infty} h(x, t)=0$. The result follows from [1] or [13].

\section{Restrictions and extensions}

We now enquire to what extent the main result, Theorem 3.5, may be extended and generalised.

The most important query concerns the restriction of small $\rho$; we first note in passing that in fact explicit bounds on $\rho$ may be found rather easily for a given $f$. The following example is instructive. In Example 2.1, take $\Omega=[0,1]$,

$$
k(x, y)= \begin{cases}-1 & (y<x) \\ 1 & (y>x) .\end{cases}
$$

and $f(u)=u\left(1-u^{2}\right)$. Linearisation about $u=0$ leads to the eigenvalue problem:

$$
\rho\left[\int_{0}^{1} k(x, y) \phi(y) d y-\phi(x)\right]+\phi(x)=\lambda \phi(x) .
$$

Differentiation of this equation yields a first order ordinary differential equation, and it is easy to show that if $\rho=1$, there are an infinite number of eigenvalue-eigenfunction pairs $\left(\lambda_{j}, \phi_{j}\right)$ where

$$
\begin{aligned}
\lambda_{j} & =\frac{2 i}{(2 j+1) \pi}, \\
\phi_{j}(x) & =e^{\sigma_{j} x},
\end{aligned}
$$

and $\sigma_{j}=2 / \lambda_{j}$.

It is clear that there is a Hopf bifurcation, and this rules out the possibility that convergence to an equilibrium holds for all $\rho>0$. 
However, it is tempting to speculate that the following may be valid.

Conjecture. Suppose $B=I$ and let $K$ be the integral operator defined as follows:

$$
K u(x)=\int_{0}^{1} k(x, y) u(y) d y,
$$

where $k$ is smooth. Then if $k>0$ on $[0,1] \times[0,1]$ and $k$ is symmetric, $L^{1}$ (and so pointwise) convergence to an equilibrium holds for all $\rho>0$ if $f$ satisfies $H 2$.

It is known [9] that the conjecture is valid in the special case $k \equiv 1$. Also, in [5] it is shown that it holds without the assumption of $k>0$ if a certain invertibility condition ensuring that all equilibria are continuous is imposed; unfortunately this condition means that the results of [5] are not applicable for small $\rho$. In the present paper we show that the result is true for very general $k$, which need be neither positive nor symmetric, but only if $\rho$ is small enough. On the other hand, the above example showing that a Hopf bifurcation may occur, demonstrates that the conjecture fails for general $k$ if $\rho$ is not restricted to be small. Thus there is some supporting evidence for the conjecture.

Our final speculation is that there is a natural extension to systems in which solutions to the reaction system (ODE) converge to equilibria. Note that the proof in the scalar case depends on the crucial condition that there is a 'gap' between equilibria, where the orbits of ODE may not remain for more than a uniformly bounded time. This result essentially follows from the purely geometrical conclusion of Lemma 3.1. This observation certainly suggests that some natural extension to systems with multistable reaction terms is possible. For example, it may be enough if for ODE the equilibria are hyperbolic and there is a Morse decomposition.

\section{References}

[1] Z. Artstein, Limiting equations and stability of nonautonomous ordinary differential equations, Appendix of J. P. Lasalle, The Stability of Dynamical Systems, SIAM, Philadelphia, PA 1976.

[2] J. Carr and R. L. Pego, Metastable patterns in solutions of $u_{t}=\varepsilon^{2} u_{x x}-f(u)$, Comm. Pure Appl. Math. 42 (1989), 523-576.

[3] E. Conway, D. Hoff, and J. Smoller, Large time behaviour of solutions of systems of non-linear reaction-diffusion equations, SIAM J. Appl. Math. 35 (1978), 1-16.

[4] D. B. Duncan, M. Grinfeld, and I. Stoleriu, Coarsening in an integro-differential model of phase transitions, Euro. J. Appl. Math. 11 (2000), 561-572.

[5] E. Feireisl, F. Issard-Roch, and Pezeltová, H. A non-smooth version of the LojasiewiczSimon theorem with applications to non-local phase-field systems, J. Diff. Eqns. 199 (2004), 1-21.

[6] P. C. Fife, An integro-differential analog of semilinear parabolic PDEs, in: Partial Differential Equations and Applications, Marcel Decker 1996, pp. 137-145. 
[7] P. C. Fife, Models for phase separation and their mathematics, in: M. Mimura and T. Nishida, eds. , Nonlinear Partial Differential Equations and Applications, Kinokuniya Pubs 2000.

[8] G. Fusco and J. K. Hale, Slow-motion manifolds, dormant instability and singular perturbations, J. Dyn. Diff. Eqns. 1 (1989), 75-94.

[9] M. Grinfeld, G. Hines, V. Hutson, K. Mischaikow, and G. Vickers, Non-local dispersal, submitted to Differential and Integral Equations (2003).

[10] K. P. Hadeler, Reaction-transport systems in biological modelling, in: Mathematics Inspired by Biology V. Capasso and O. Diekmann, eds., Lecture Notes in Mathematics 1714, Springer-Verlag, New York 1999.

[11] V. Hutson, S. Martinez, K. Mischaikow, and G. T. Vickers, The evolution of dispersal. J. Math. Biol. 47 (2003), 483-517.

[12] H. Matano, Asymptotic behaviour and stability of solutions of semilinear diffusion equations, Publ. RIMS, Kyoto Univ. 15 (1979), 401-454.

[13] K. Mischaikow, H. Smith, and H. Thieme, Asymptotically autonomous semiflows; chain recurrence and Lyapunov functions, Trans. AMS 347 (1995), 1669-1685.

[14] J. D. Murray, Mathematical Biology, II Spatial Models and Biomedical Applications, 3rd edition, Springer-Verlag, New York 2003.

[15] H. G. Othmer, S. R. Dunbar, and W. Alt, Models of dispersal in biological systems, J. Math. Biol. 26 (1988), 263-298.

[16] P. Poláčik, Parabolic equations: asymptotic behaviour and dynamics on invariant manifolds, in: Handbook of Dynamical Systems, Vol. II, Section D, Elsevier Science, Amsterdam 2002.

[17] I. Stoleriu, Integro-Differential Equations in Materials Science, $\mathrm{Ph}$. D. Thesis, University of Strathclyde, Glasgow 2001. 